\title{
Gapless Superconductivity
}

\author{
Boris V. Bondarev ${ }^{*}$ \\ Moscow Aviation Institute, VolokolamskoeShosse, 4, 125871, Moscow, Russia \\ *Corresponding author: bondarev.b@mail.ru
}

Received May 23, 2014; Revised February 10, 2015; Accepted February 25, 2015

\begin{abstract}
The mean field method is applied for analysis of valence electrons in metals. It is shown that at low temperatures electrons have two wave-vector distribution patterns. Isotropic distribution refers to the first pattern. Anisotropic distribution refers to another pattern, particularly to specific wave vector values occurred nearby the Fermi sphere. It is shown that it is the anisotropy that makes the metal obtain its specific superconductor features.
\end{abstract}

Keywords: valence electrons, mean field method, Fermi-Dirac function, isotropic and anisotropic distributions, superconductivity

Cite This Article: Boris V. Bondarev, "Gapless Superconductivity.” International Journal of Physics, vol. 3, no. 2 (2015): 88-95. doi: 10.12691/ijp-3-2-7.

\section{Introduction}

Superconductivity was discovered by KamerlinghOnnes in 1911 in the Netherlands in Leiden laboratory [1]. Studying the dependence of the resistance on the temperature of mercury, he found that with decreasing temperature of about $4 \mathrm{~K}$ resistance suddenly vanishes. This phenomenonis called superconductivity. Shortly have found other elements having these properties. Superconductivity got its theoretical explanation in 1950 at the phenomenological level in the Ginzburg-Landau [2] and in 1957 at the microscopic level in the BardeenCooper-Schrieffer [3,4]. Recently opened other types of superconductivity [5,6]. In this paper we propose another microscopic theory of superconductivity [7-11].

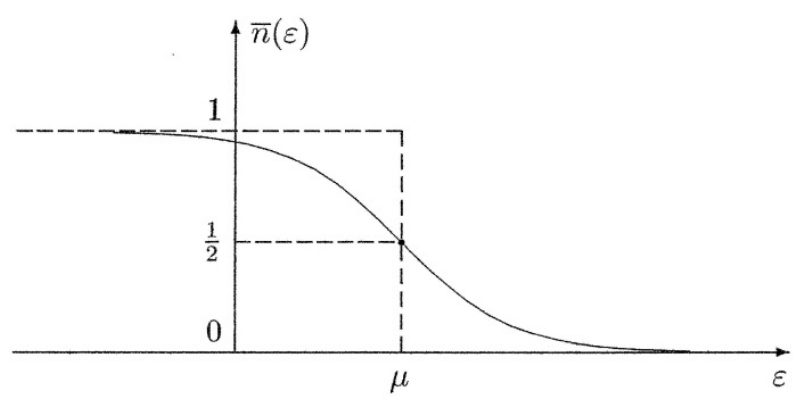

Figure 1. Fermi-Dirac Function

Consider how to construct a function of the FermiDirac and what it makes sense [12]. First, it is mainly about electrons, which are very, very much in the solid. Second, the electrons obey the Pauli exclusion principle, according to which a state can not be more than one electron. The simplest form of this function such

$$
\bar{n}(\varepsilon)=\frac{1}{\mathrm{e}^{\beta(\varepsilon-\mu)}+1},
$$

here $\bar{n}(\varepsilon)$ - average number of electrons in a state with energy $\varepsilon, \beta=1 / k_{\mathrm{B}} T^{-}$inverse temperature, $\mu$-chemical potential. The form of this function is shown in Figure 1.

Function Fermi-Dirac strictly output only for a system of non-interacting electrons. If the electrons interact, it is possible to derive an equation for the function $\bar{n}(\varepsilon)$ only in some approximation. The simplest approximation is the mean-field approximation, in which it is considered that the double probability is equal to the product of the single probabilities. This approximation is called the statistical independence. In the mean-field approximation equation is

$$
\bar{n}(\varepsilon)=\frac{1}{\mathrm{e}^{\beta(\bar{\varepsilon}-\mu)}+1}
$$

is $\bar{\varepsilon}$ the mean electron energy, which is equal to the kinetic energy $\varepsilon$ and the interaction energy of the electron with other electrons.

Now remember that some representation in state of the electron is described by a wave function $\psi_{\mathbf{k} n}(\mathbf{r}) \chi_{\sigma}(\xi)$, where $\mathbf{k}$ - the wave vector, $n$ and $\sigma$-other numbers that together with the wave vector determine the state of the electron. Wave vector associated with the electron momentum simple relation: $\mathbf{p}=\hbar \mathbf{k}$. We now write, without going into details, the equation (1.2) in the form

$$
\ln \frac{1-w_{\mathbf{k}}}{w_{\mathbf{k}}}=\beta\left(\bar{\varepsilon}_{\mathbf{k}}-\mu\right)
$$

where $w_{\mathbf{k}}=\bar{n}\left(\varepsilon_{\mathbf{k}}\right)$ is the probability electron state with wave vector $\mathbf{k}, \bar{\varepsilon}_{\mathbf{k}}$ - the average energy of an electron, which is equal to the kinetic energy of the electron $\varepsilon_{\mathbf{k}}$ and the average energy of interaction of the electron with other electrons: 


$$
\bar{\varepsilon}_{\mathbf{k}}=\varepsilon_{\mathbf{k}}+\sum_{k^{\prime}} \varepsilon_{\mathbf{k} \mathbf{k}^{\prime}} w_{\mathbf{k}^{\prime}}
$$

where the term $\varepsilon_{\mathbf{k k}}$ is the energy of interaction of two electrons with wave vectors $\mathbf{k}$ and $\mathbf{k}$

It can be shown that the Coulomb interaction energy of two electrons in a solid under certain conditions, has the form [7-11]

$$
\varepsilon_{\mathbf{k} \mathbf{k}^{\prime}}=I \delta_{\mathbf{k}+\mathbf{k}^{\prime}}-J \delta_{\mathbf{k}-\mathbf{k}^{\prime}}
$$

Here $I$ is the repulsion energy electrons with wave vectors equal in magnitude and opposite in direction: $\mathbf{k}=-\mathbf{k}^{\prime}, J$ - the energy of attraction between two electrons in states with equal wave vectors: $\mathbf{k}=\mathbf{k}^{\prime}, \delta_{\mathbf{k}}$ Kronecker delta.

\section{Anisotropy}

Let us now consider what the anisotropic distribution [13]. We have prescribed function $f=f(a)$, i.e. value $f$ depends on vector a. If value $f$ depends on modulus a of this vector only, the distribution concerned is called isotropic, i.e. it may be formulated as $f=f(a)$. This kind of isotropy may be represented graphically (see Figure 2). We will plot a sphere of radius a centered in the origin of coordinates. So, value $f$ will remain equal at any point of this sphere, providing that $f=f(a)$ is the isotropic function. Any other $\mathrm{f}=\mathrm{f}(\mathbf{a})$ function will be referred to the anisotropy one.

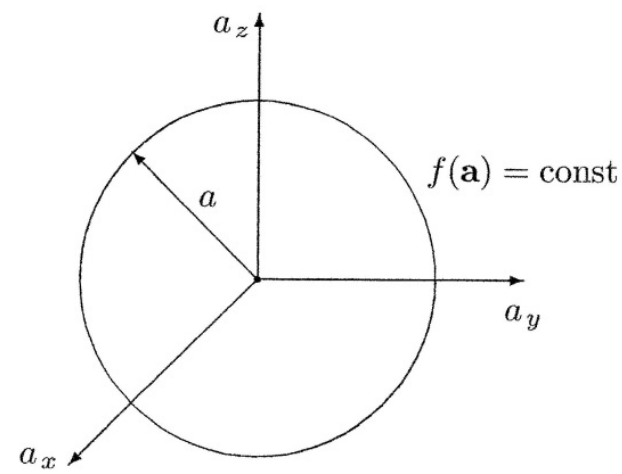

Figure 2. Isotropic function

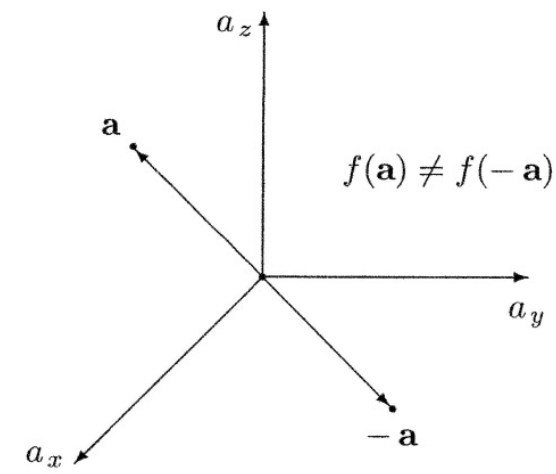

Figure 3. Example of anisotropic function

Now, we will consider the example of the anisotropic function. We will plot two vectors. One of them will be an arbitrary vector a and the other one will be rated as equal, but opposite in its direction -a. Two such vectors are shown in Figure 3. So, if it is appeared that function values fail matching in the points concerned, i.e. $\mathrm{f}(\mathbf{a}) \neq \mathrm{f}(--$ a), this function will be called the anisotropic one. Some exhaustive examples of the anisotropic function may be additionally described, but, as a matter of fact, the information provided is sufficient for understanding.

\section{The Model Hamiltonian}

Equation (1.3) contains its non-linear manner unknown function $w_{\mathbf{k}}$. Now to find this feature, you need to know the energy of the interaction of electrons $\varepsilon_{\mathbf{k k}}$ ' Electrons interact with each other by Coulomb repulsion potential. But solid superimposed on the interaction of electrons and ions of a large number of other electrons. It is a crystal lattice plays a crucial role in the interaction energy of the electrons. Calculation leads to the formula (1.5). For simplicity, in this formula, $J=0$. This inequality means that the electrons do not form a pair. Therefore, we will talk about gapless conduction. Obtain a simpler model Hamiltonian

$$
\varepsilon_{\mathbf{k} \mathbf{k}^{\prime}}=I \delta_{\mathbf{k}+\mathbf{k}^{\prime}} \cdot
$$

As provided in scope of our model, only those valence electrons are repulsive, which are able to surmount the crystal area at equal opposite direction velocities.

With the model formula applied (3.1) the average electron energy (1.4) is formulated by the equation as follows:

$$
\bar{\varepsilon}_{\mathbf{k}}=\varepsilon_{\mathbf{k}}+I w_{-\mathbf{k}}
$$

According to this formula, the energy of the electron with the wave vector $\mathbf{k}$ is smaller, the less likely the $w_{-\mathbf{k}}$ filling state with wave vector $-\mathbf{k}$. Thus, electron with wave vector -k as it displaces an electron from a state with a wave vector $\mathbf{k}$.

We will insert formula (3.2) in equation (1.3). We will gain the following formula:

$$
\ln \frac{1-w_{\mathbf{k}}}{w_{\mathbf{k}}}=\beta\left(\varepsilon_{\mathbf{k}}+I w_{-\mathbf{k}}-\mu\right)
$$

Thus, the equation containing two function values $w_{\mathbf{k}}$ and $w_{-\mathbf{k}}$ is produced.

\section{Isotropic and Anisotropic Electron Distribution}

If you ask why it has to do with anisotropy, it may be confirmed by the equation (3.3) which exhibits solution of anisotropic function $w_{\mathbf{k}}$ subject to condition of $w_{-\mathbf{k}} \neq w_{\mathbf{k}}$. In this equation we will substitute vector $\mathbf{k}$ for vector $\mathbf{- k}$. If to consider that kinetic energy is the isotropic function, i.e. $-\varepsilon_{\mathbf{k}}=\varepsilon_{\mathbf{k}}$, we will formulate the following equation:

$$
\ln \frac{1-w_{-\mathbf{k}}}{w_{-\mathbf{k}}}=\beta\left(\varepsilon_{\mathbf{k}}+I w_{\mathbf{k}}-\mu\right)
$$


Equations (3.3) and (4.1) produce the system containing two unknown $w_{\mathbf{k}}$ and $w_{-\mathbf{k}}$. At the same time, it is clear enough that probability $w_{\mathbf{k}}$ is the composite $\mathbf{k}$ vector function, where electron kinetic energy is applied as an intervening variable $\varepsilon_{\mathbf{k}}$ :

$$
w_{\mathbf{k}}=w\left(\varepsilon_{\mathbf{k}}\right)
$$

Combined equations (3.3) and (4.1) exhibit their anisotropic solution subject to the condition as follows:

$$
w_{-\mathbf{k}}=w_{\mathbf{k}}
$$

Using this equation we will eliminate $w_{-\mathbf{k}}$ from the combined equations (3.3) and (4.1). We will find solution of isotropic distribution function by applying the following equation:

$$
\ln \frac{1-w_{\mathbf{k}}}{w_{\mathbf{k}}}=\beta\left(\varepsilon_{\mathbf{k}}+I w_{\mathbf{k}}-\mu\right)
$$

There are also some anisotropic distribution functions that fall out of formula (4.3) when specific wave vector values are applied:

$$
w_{-\mathbf{k}}=w_{\mathbf{k}}
$$

Such kind of electron state distribution anisotropy may occur even when no external field is available. While applying forms:

$$
w_{-\mathbf{k}}=w_{1}\left(\varepsilon_{\mathbf{k}}\right), w_{\mathbf{k}}=w_{2}\left(\varepsilon_{\mathbf{k}}\right)
$$

We may formulate equations (3.3) and (4.1) by the method as follows:

$$
\ln \frac{1-w_{1}}{w_{1}}=\frac{4}{\tau}\left(\epsilon+w_{2}\right), \ln \frac{1-w_{2}}{w_{2}}=\frac{4}{\tau}\left(\epsilon+w_{1}\right)
$$

where

$$
\epsilon=\frac{\varepsilon-\mu}{I}, \tau=\frac{4 \theta}{I}
$$

The following functions remain unknown in the combined equations (4.6):

$$
w_{1}=w_{1}(\varepsilon) \text { and } w_{2}=w_{2}(\varepsilon)
$$

If electrons have isotropic wave vector distribution, it is necessary to insert $w_{1}=w_{2}=w_{0}$ in the combined equation (4.6). In this case, the equation gained may be formulated by the method as follows:

$$
\epsilon=\frac{\tau}{4} \ln \frac{1-w_{0}}{w_{0}}-w_{0}
$$

This equation states specific dependence of $w_{0}=w_{0}(\varepsilon)$ with various temperature values graphically represented in Figure 4 in the form of monotonically decreasing curves.

If electrons have anisotropic wave vector distribution, probabilities $w_{1}$ and $w_{2}$ in the combined equations (4.6) shall be considered as various functions $\epsilon: w_{1}=w_{1}(\epsilon)$ and $w_{2}=w_{2}(\epsilon)$ subject to energy. To determine these dependences, we will introduce new variables $d$ and $s$ applying the relations as follows:

$$
\begin{aligned}
& w_{2}-w_{1}=d, \\
& w_{1}+w_{2}=1+s .
\end{aligned}
$$

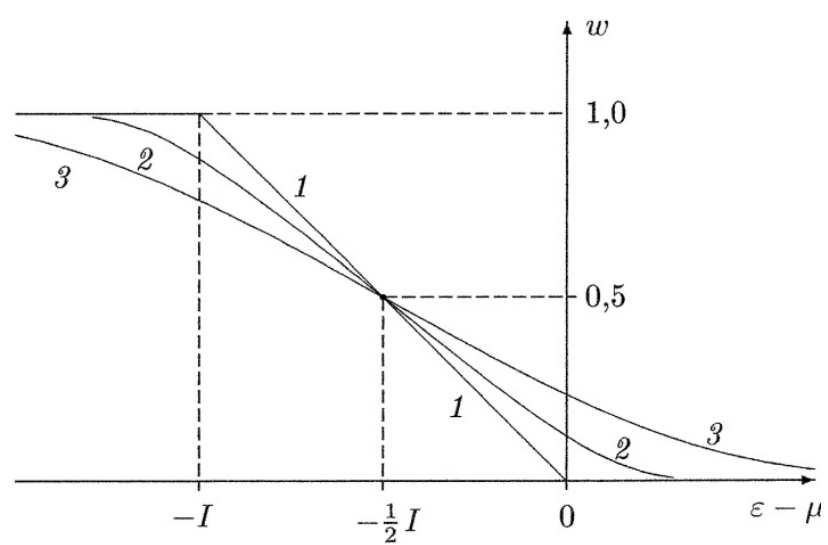

Figure 4. Isotropic function of distribution of conductivity electron energy at various temperature values: $1-\tau=0 ; 2-\tau=0,25 ; 3-\tau=0,8$

Without loss of generality we will assume that nonnegative difference $d$ of two distribution function values $w_{1}$ and $w_{2}$ is $d \geq 0$. At the same time, $d$ remains equal to $d \in[0,1]$. Value $s$ may possess the values within the range of -1 to $1: s \in[-1,1]$. We will determine the equalities (4.8), as regards the probabilities $w_{1}$ and $w_{2}$ :

$$
w_{1}=\frac{1}{2}(1+s-d), w_{2}=\frac{1}{2}(1+s+d)
$$

We will transform the combined equations (4.6) by applying the formulas (4.9). We will firstly subtract specific equation from one of the combined equations and then we will add the equations. As a result, we will obtain the following combined equations:

$$
\begin{aligned}
& \frac{(1+d)^{2}-s^{2}}{(1-d)^{2}-s^{2}}=\mathrm{e}^{4 d / \tau} \\
& \epsilon=\frac{\tau}{8} \ln \frac{(1-s)^{2}-d^{2}}{(1+s)^{2}-d^{2}}-\frac{1+s}{2}
\end{aligned}
$$

The first equation of the combined ones may be easily resolved against $s$ :

$$
s(d)= \pm \sqrt{\frac{(1-d)^{2} \mathrm{e}^{4 d / \tau}-(1+d)^{2}}{\mathrm{e}^{4 d / \tau}-1}}
$$

As concerns the relations (4.9), probabilities $w_{1}$ and $w_{2}$ may be also considered as $d$ functions:

$$
w_{1}=w_{1}(d), w_{2}=w_{2}(d) .
$$

With the second equation of the combined ones (4.10) applied, we may express $\epsilon$ electron energy in terms of parameter $d$. Using the dependences produced specific graphs of functions

$$
w_{1}=w_{1}(\epsilon), w_{2}=w_{2}(\epsilon) .
$$

may be easily plotted for various temperature values. For the plotted curve, see Figure 5. 


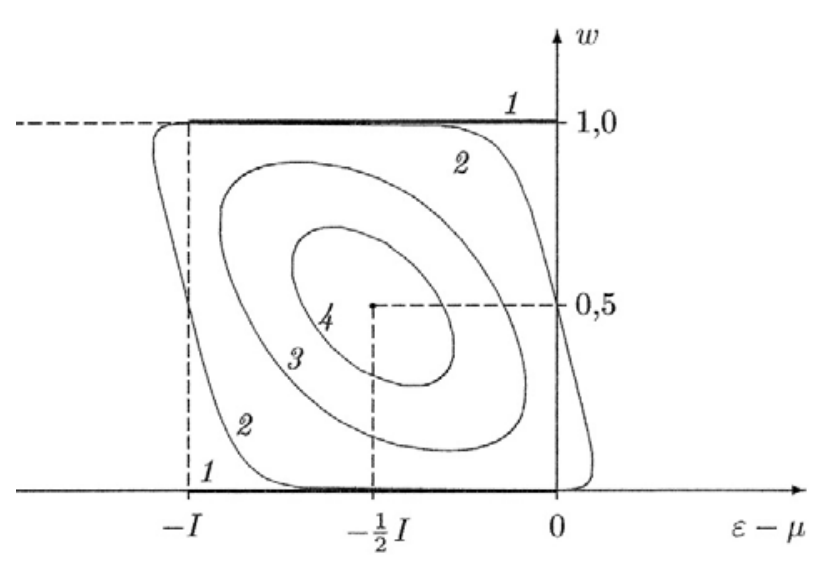

Figure 5. Anisotropic function of distribution of conductivity electron energy at various temperature values: $1-\tau=0 ; 2-\tau=0,25 ; 3-\tau=0,8 ; 4$ $\tau=0,95$

The pattern of distribution of electrons by their states depends on their relation between metal temperature $T$ and critical temperature:

$$
T_{\mathrm{C}}=\frac{I}{4 k_{\mathrm{B}}} .
$$

At the temperature of $T \geq T_{\mathrm{C}}$ the distribution function $w=w(\epsilon)$ is single-valued and satisfies the condition (4.3), as respects all the $\epsilon$ energy values. At the temperature of $T<T_{\mathrm{C}}$ the energy is limited by $\left(\epsilon_{1}, \epsilon_{2}\right)$ with function $w=w(\epsilon)$ possessing any of three values at every point of the limit, particularly $w_{1}<w_{0}<w_{2}$. Being out of the aforesaid limit, the distribution function $w=w(\epsilon)$ possesses only a single value $w_{0}(\epsilon)$. Thus, equation (4.4) is resolved by applying function $w_{\mathbf{k}}=w_{0}\left(\epsilon_{\mathbf{k}}\right)$ to describe isotropic wave vector electron distribution.

\section{Order Parameter}

At $T<T_{\mathrm{C}}$ some kind of anisotropic wave vector electron distribution may occur in the narrow layer Sunder Fermi surface $\varepsilon_{\mathbf{k}}=\mu$. This kind of distribution is formulated by

$$
\begin{aligned}
& w_{\mathbf{k}}=w_{2}\left(\epsilon_{\mathbf{k}}\right), \\
& w_{-\mathbf{k}}=w_{1}\left(\epsilon_{\mathbf{k}}\right)
\end{aligned}
$$

subject to $\epsilon \in\left(\epsilon_{1}, \epsilon_{2}\right)$. Difference $d=w_{2}-w_{1}$ of two anisotropic electron distribution function values possesses the largest value $d_{\max }$ subject to $\epsilon=0,5$. In this case, $w_{0}=0,5$ and $s=0$. We will determine difference $d_{\max }$ from temperature $\tau$ by applying $s=0$ in the first equation (4.10):

$$
\frac{2 d_{\max }}{\tau}=\ln \frac{1+d_{\max }}{1-d_{\max }}
$$

For dependence curve, see Figure 6.

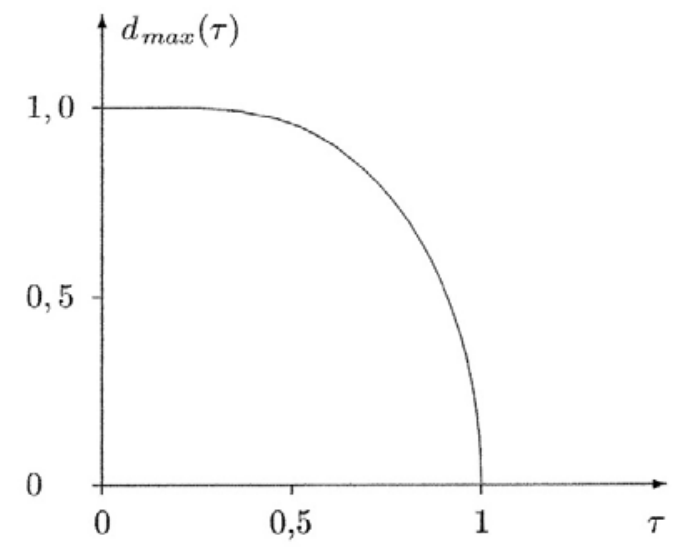

Figure 6. Electron distribution anisotropy parameter $d_{\max }$, as $\tau$ temperature function

\section{Electron $T=0$ Distribution}

At $T=0$ the isotropic distribution function is formulated as follows:

$$
\begin{aligned}
& 1 \text { at } \varepsilon_{\mathbf{k}} \leq \mu-I, \\
& w_{\mathbf{k}}=-\frac{1}{I}\left(\varepsilon_{\mathbf{k}}-\mu\right) \text { at } \mu-I<\varepsilon_{\mathbf{k}}<\mu, \\
& 0 \text { at } \varepsilon_{\mathbf{k}} \geq \mu
\end{aligned}
$$

As for the anisotropic distribution, it is formulated as follows:

$$
\begin{aligned}
& w_{\mathbf{k}}=1 \text { at } \varepsilon_{\mathbf{k}} \leq \mu-I, \\
& w_{\mathbf{k}}=1, w_{-\mathbf{k}}=0 \text { or } w_{\mathbf{k}}=0, w_{-\mathbf{k}}=1 \\
& \text { at } \mu-I<\varepsilon_{\mathbf{k}}<\mu, w_{\mathbf{k}}=0 \text { at } \varepsilon_{\mathbf{k}} \geq \mu .
\end{aligned}
$$

As provided by the formula (6.2), layer $\mathrm{S}$ may be determined under Fermi surface by the inequality (6.3), in which the electrons have anisotropic wave vector distribution, i.e. one of the both $\mathbf{k}$ and $-\mathbf{k}$ wave vector states in this layer is free and another one is occupied. For the function curves, see Figure 7. Apparently, electron distribution function obtains its three $\mathrm{S}$ layer values. And what is the matter it stands for? The answer is in the value of energy, the isotropic or anisotropic distribution electrons exhibit. The electrons gain their steady state when they have the lowest energy.

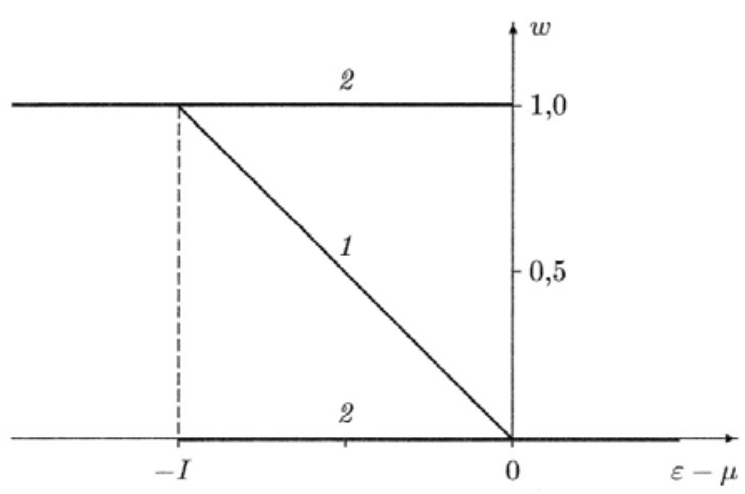

Figure 7. Isotropic and anisotropic distribution of conductivity electrons depending on their kinetic energy at temperature $\tau=0$ : 1 - isotropic distribution, 2 - anisotropic distribution 


\section{Superconductivity. Energy of States}

In scope of normalization conditions, the average itinerant electron velocity may be defined by the formula as follows:

$$
v=\frac{\mathrm{G} \hbar}{\mathrm{m} \overline{\mathrm{N}}} \sum_{k} \mathbf{k} w_{\mathbf{k}}
$$

where $G=2 n_{s}$ is a number of states in one node and $\bar{N}-$ mean number of conductivity electrons in a crystal. If the distribution function is isotropic, mean electron velocity $\mathbf{v}$ gets equal to zero. Formula (7.1) may assign specific nonzero electron ordered motion velocity values to some anisotropic distribution functions, i.e. these distribution functions are applicable for defining electric current. If there are steady-state currents to exist with no external fields available, than such itinerant electron system states shall be considered as the superconductive ones [5,6,7].

We will assume that the state of electron gas is described by the anisotropic distribution function (5.1) or (6.2). In this case, mean electron ordered motion velocity modulus $v$ may assign any value rated from zero to certain $v_{\max }$. The mean velocity will be equal to zero, providing that free pairs and those occupied by wave vectors $\mathbf{k}$ and $\mathbf{k}$ are chaotically distributed within layer S. If all the states concerned are occupied in one half of the layer (this is to say at $k_{x}>0$ ) and free in another half of the layer (at $k_{x}<0$ ), the electrons will gain their maximum ordered motion velocity. The value assigned by the mean electron velocity is defined by the nature of initial electron gas state. If the pattern of anisotropic wave vector electron distribution is rather steady with respect to small environment variations, the electron velocity value will survive for ages. This means that the metal concerned was able to gain its specific superconductive characteristics.

Now, we will calculate the energy the isotropic and anisotropic distribution electrons exhibit. We will apply the normalization condition formulated as follows:

$$
G \sum_{k} w_{\mathbf{k}}=\overline{\mathrm{N}}
$$

Mean field approximation electron energy takes on the following form:

$$
\bar{E}=G \sum_{\mathbf{k}}\left(\varepsilon_{\mathbf{k}} w_{\mathbf{k}}+\frac{1}{2} I w_{\mathbf{k}} \mathrm{w}_{-\mathbf{k}}\right)
$$

We will approximate dependence of electron kinetic energy $\varepsilon_{\mathbf{k}}$ from wave vector $\mathbf{k}$ by applying the formula as follows:

$$
\varepsilon_{\mathbf{k}}=\frac{\hbar^{2} k^{2}}{2 m},
$$

where $m$ is effective itinerant electron mass. As provided by this formula, any electron kinetic energy shall be counted from the band bottom to be also called "bottom of conduction band" i.e. $\varepsilon_{\mathbf{k}=0}=0$.

To simplify calculations, instead of $\mathbf{k}$ summing we will produce integration by $\varepsilon$ electron kinetic energy. By applying the dependence (7.4) we will obtain the following symbolic equation:

$$
G \sum_{\mathbf{k}} \ldots=A(\overline{\mathrm{N}}) \int_{0}^{\infty} \ldots \sqrt{\varepsilon} \mathrm{d} \varepsilon
$$

where

$$
A=\frac{G m \sqrt{2 m} V}{2 \pi^{2} \hbar^{3} \bar{N}}, \varepsilon=\frac{\hbar^{2} k^{2}}{2 m}, \mathrm{~d} \varepsilon=\frac{\hbar^{2} k \mathrm{~d} k}{m} .
$$

The upper integration limit may be equal to $\infty$, since the occupational probability of states which energy $\varepsilon$

is specified at the ceiling of conduction band is actually equal to zero. Now, we will formulate the normalization condition (7.2) by the method as follows:

$$
\mathrm{A} \int_{0}^{\infty} w(\epsilon) \sqrt{\varepsilon} \mathrm{d} \varepsilon=1
$$

As for the isotropic distribution electron energy, we will formulate the following formula:

$$
\bar{E}^{-(i)}=A \bar{N} \int_{0}^{\infty}\left(\varepsilon+\frac{1}{2} I w(\epsilon)\right) w(\epsilon) \sqrt{\varepsilon} \mathrm{d} \varepsilon,
$$

If the isotropic electron distribution function applied at $T=0$ is formulated according to (6.1), the equations (7.5) and (7.6) take on the following form:

$\mathrm{A} \int_{0}^{\mu-1} \sqrt{\varepsilon} d \varepsilon+A \int_{\mu-I}^{\mu} \frac{\mu-\varepsilon}{I} \sqrt{\varepsilon} d \varepsilon=1$,

$\bar{E}_{0}^{(i)}=A \bar{N} \int_{0}^{\mu-I}\left(\varepsilon+\frac{1}{2} I\right) \sqrt{\varepsilon} \mathrm{d} \varepsilon+\frac{\mathrm{A} \bar{N}}{2 I} \int_{\mu-I}^{\mu}\left(\mu^{2}-\varepsilon^{2}\right) \sqrt{\varepsilon} d \varepsilon$.

Since we apply the small parameter

$$
\lambda=\frac{I}{\varepsilon_{F}}
$$

where

$$
\varepsilon_{F}=(3 / 2 \mathrm{~A})^{2 / 3}
$$

refers to Fermi energy, we will define that the chemical potential and isotropic wave vector distribution electron energy at $T=0$ take on the forms as follows:

$$
\begin{aligned}
& \mu_{0}=\varepsilon_{F}\left(1+\frac{1}{2} \lambda-\frac{1}{48} \lambda^{2}+\ldots\right) \\
& \bar{E}_{0}(i)=-\bar{N} \varepsilon_{F}\left(\frac{3}{5}+\frac{1}{2} \lambda-\frac{1}{16} \lambda^{2}+\ldots\right) .
\end{aligned}
$$

We will assume that the anisotropic wave vector electron distribution at $T=0$ is defined by the function as follows:

$$
\begin{aligned}
& =1 \text { at } \varepsilon_{\mathbf{k}} \leq \mu-I, \\
& =1 \text { at } \mu-I<\varepsilon_{\mathbf{k}}<\mu, k_{x}>0, \\
& w_{\mathbf{k}}=0 \text { at } \mu-I<\varepsilon_{\mathbf{k}}<\mu, k_{x}<0, \\
& =0 \text { at } \varepsilon_{\mathbf{k}} \geq \mu .
\end{aligned}
$$

As provided by the above formula, only one half of the $k_{x}>0$ states may be referred to as the occupied ones to occur in layer $\mathrm{S}$ above the Fermi surface, which thickness $\delta \mathbf{k}$ is proportional to interaction parameter I. For anisotropic distribution pattern, see Figure 8. 


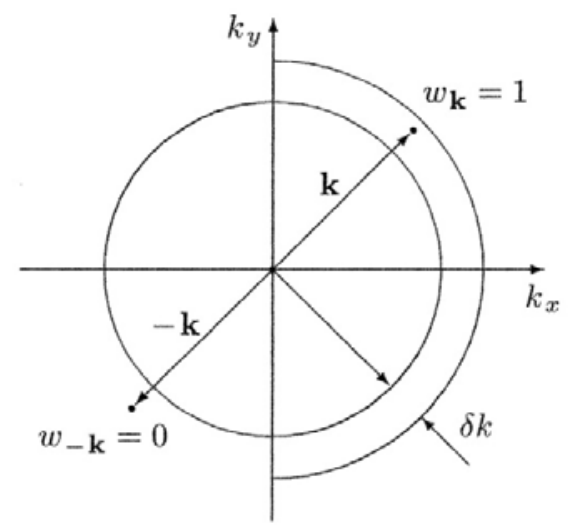

Figure 8. Anisotropic distribution function at $T=0$

Here, the normalization condition gives rise to the following equation:

$$
\mathrm{A} \int_{0}^{\mu-1} \sqrt{\varepsilon} d \varepsilon+\frac{1}{2} A \int_{\mu-I}^{\mu} \frac{\mu-\varepsilon}{I} \sqrt{\varepsilon} d \varepsilon=1,
$$

As for electron energy, it may be calculated by the formula as follows:

$$
\bar{E}_{0}^{(s)}=A \bar{N} \int_{0}^{\mu-I}\left(\varepsilon+\frac{1}{2} I\right) \sqrt{\varepsilon} \mathrm{d} \varepsilon+\frac{\mathrm{A} \bar{N}}{2} \int_{\mu-I}^{\mu} \varepsilon \sqrt{\varepsilon} d \varepsilon .
$$

As provided by the above calculation, the following formulation is obtained:

$$
\begin{aligned}
& \mu_{0}=\varepsilon_{F}\left(1+\frac{1}{2} \lambda-\frac{1}{16} \lambda^{2}+\ldots\right), \\
& { }_{-}^{(s)}=-\bar{N} \varepsilon_{F}\left(\frac{3}{5}+\frac{1}{2} \lambda-\frac{3}{16} \lambda^{2}+\ldots\right) .
\end{aligned}
$$

If occupied and free state pairs that match specific wave vectors $\mathbf{k}$ and--k will be distributed within layer S by any other way, the chemical potential and electron energy rating will remain the same.

The difference of electron energy values (7.7) and (7.9) will be formulated by the equation as follows:

$$
\bar{E}_{0}^{(i)}-\bar{E}_{0} \cong \frac{\bar{N} I^{2}}{8 \varepsilon_{F}}>0 .
$$

Thus, we get to the conclusion that the state of itinerant electrons described by the anisotropic distribution function is the primary one -- i.e. the electron system specified in this condition is of the lowest energy.

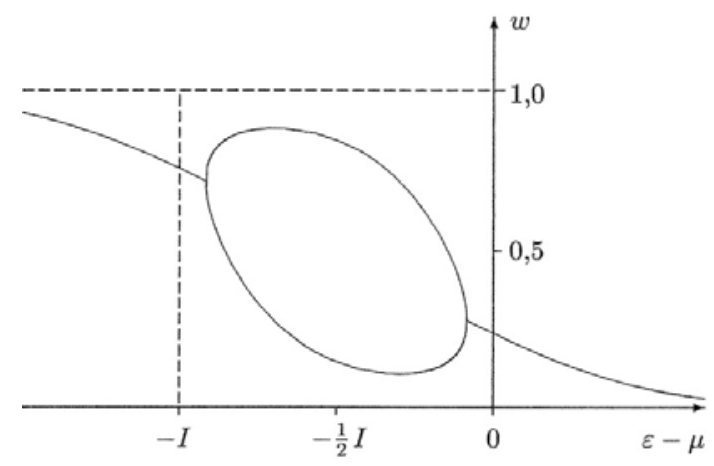

Figure 9. Anisotropic distribution of energy $\varepsilon$ conductivity electrons subject to the lowest energy $E$ at the temperature of $\tau=0,8$
Considering for the aforesaid about the anisotropic electron energy distribution we will plot the pattern of superconductive state, as shown in Figure 9.

\section{Maximum Superconductivity Electron Velocity at $T=0$}

Now, we will find ordered electron motion velocity in the state described by the distribution function (6.2) at the temperature of $T=0$. For this purpose, we well substitute the wave vector sum specified in the formula (7.1) for the following integral:

$$
\mathbf{v}=\frac{G \hbar \mathrm{V}}{8 \pi^{3} m \frac{-}{N}} \int \mathbf{k} w_{\mathbf{k}} \mathbf{d}^{3} \mathbf{k}
$$

If $T=0$, the inner and outer radii of layer $\mathrm{S}$ shall be respectively equal to as follows:

$$
k_{1}=\frac{1}{\hbar} \sqrt{2 m(\mu-I)}, k_{2}=\frac{1}{\hbar} \sqrt{2 m \mu}
$$

In this case, the layer thickness is defined by the following formula:

$$
\mathrm{d} k=k_{2}-k_{1}=\frac{I}{\hbar} \sqrt{\frac{m}{2 \varepsilon_{F}}} .
$$

If the states are filled with electrons in one half of layer $\mathrm{S}$, but another one remains free, the rate of ordered electron motion velocity exhibits its maximum value

$$
v_{\max }=\frac{3 I}{4 \sqrt{2 m \varepsilon_{F}}} .
$$

\section{Electric Current-Forced Superconduc- ting State Destroy Effect. Silsbee Effect}

Superconducting state of the itinerant electrons is destroyed in the events when the current running over metal items exceeds its particular critical value (Silsbee effect). We will assume that specific homogeneous electric field with tension $\mathbf{E}$ is produced inside the metal. Under the effect of this field the itinerant electrons will execute their ordered motion at the average velocity $\mathbf{u}$ which direction agrees with another one that affecting force electron - eE. In this case, electron state distribution function $w_{\mathbf{k}}$ may be defined by the equation (1.3), where electron energy $\bar{\varepsilon}_{\mathbf{k}}$ depends on wave vector $\mathbf{k}$ in the following manner:

$$
\bar{\varepsilon}_{\mathbf{k}}=\varepsilon_{\mathbf{k}}-\hbar \mathbf{k} \mathbf{u}+I w_{-\mathbf{k}}
$$

It is no easy matter to make exact solution of the equation (1.3). Therefore, we will consider its approximate solution only. We will assume that distribution function $w_{\mathrm{k}}$ has the form as follows:

$$
w_{\mathbf{k}}=f\left(\mathbf{k}-\mathbf{k}_{0}\right)
$$

Where $w_{\mathbf{k}}=f(\mathbf{k})$ is the solution of the equation (1.3), providing that $\mathbf{u}=0$; 


$$
\mathbf{k}_{0}=\frac{m \mathbf{u}}{\hbar}
$$

Function $w_{\mathbf{k}}=f(\mathbf{k})$ is equal to a unity almost at all the points occurred inside Fermi sphere: $k<k_{F}$, excluding the points at surface $S$ of that sphere. If occurred outside Fermi sphere, function $w_{\mathbf{k}}=f(\mathbf{k})$ almost everywhere is equal to zero. Field D containing nonzero function (9.2) is limited by the sphere with radius $k_{F}$, which center $\mathrm{C}$ is displaced off the origin of coordinates $\mathrm{O}$ by vector $\mathbf{k}_{0}$. In other words, the wave vectors that agree with occupied electronic states belong to field D. The theory under discussion is applied to superconductivity to be due to interaction of electrons, which wave vectors $\mathbf{k}$ and $-\mathbf{k}$ belong to spherical layer $\mathrm{S}$ with its radius equal to $k_{F}$ and thickness - to $\delta k$. No anisotropy is created with the electrons distributed over their wave vectors, when the rate of displacement $\mathbf{k}_{0}$ of field D is so large that one half of layer $S$ finds itself beyond this field. For arrangement of field D and layer S, see Figure 10.

Point A belongs simultaneously to the displaced Fermi sphere and to the inner surface of layer S. Therefore,

$$
\mathrm{AC}=k_{F}, \mathrm{AO}=k_{F}-\delta k .
$$

We will apply the rule of Pythagoras for AOC triangle. Now, we will gain the following formula:

$$
k_{F}^{2}=k_{0}^{2}+\left(k_{F}-\delta k\right)^{2}
$$

It appears from this equation that superconductivityforce displacement $k_{0}$ will be formulated as follows:

$$
k_{0}=\sqrt{2 k_{F} \delta k}
$$

This formula may be transformed to:

$$
k_{0}=\frac{\sqrt{2 m \delta \varepsilon}}{\hbar},
$$

Where $\delta \varepsilon$ is energy width of the layer $\mathrm{S}$.

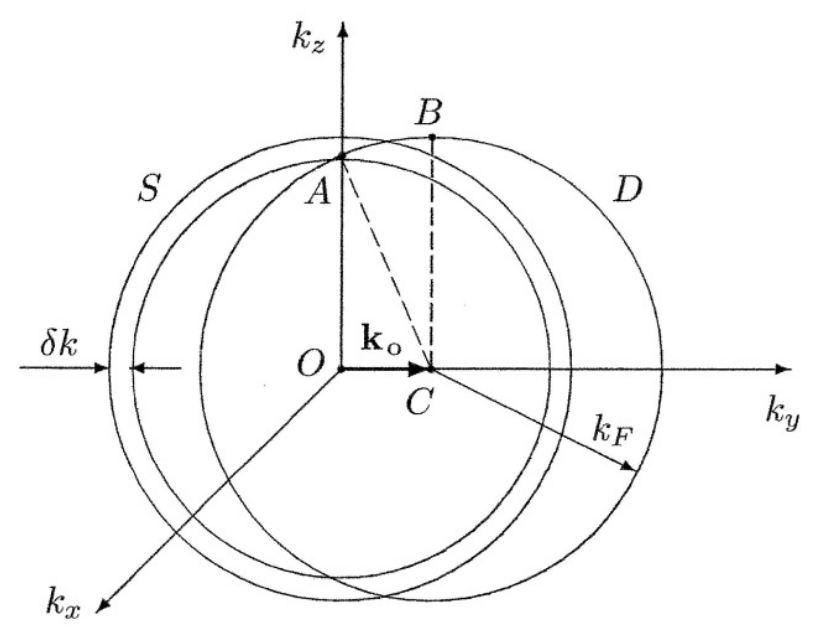

Figure 10. Displacement of Fermi sphere under influence of electric field

In this connection, the average current velocity will be calculated by the following equation:

$$
u=\sqrt{\frac{2 \delta \varepsilon}{m}}
$$

By this means that the superconductive state of conduction electrons will be destroyed when the external electric field makes them moving to the same direction and produces the current, which density exceeds the following value:

$$
j_{c r}=\mathrm{enu}
$$

We will specify the relation of maximum superconductive current velocity $v_{\max }$ to the least current speed $u$ destroying the superconductive state:

$$
\frac{v_{\max }}{u}=\frac{3}{8} \sqrt{\frac{\delta \varepsilon}{\varepsilon_{F}}}
$$

It is clear enough that the above relation is much less than unity $-v_{\text {max }} \ll u$.

On cutting off the electric field and after thermodynamic equilibration the electrons shall transform to their superconductive state distributing as provided by the formula (7.2). Thereafter, the average electron velocity shall drop down to value $v_{\max }$ and superconductive current of maximum density $j_{\max }$ will run over the metal.

\section{Mean Energy Dependence of Kinetic Energy}

The rate of mean electron $\bar{\varepsilon}_{\mathbf{k}}$ energy dependence of its kinetic energy $\varepsilon_{\mathbf{k}}$ is defined by formula (3.2). As may be inferred from the above formula, the electron energy with wave vector $\mathbf{k}$ depends on whether the -k wave vector state is free or occupied. Electron energy $\bar{\varepsilon}$ may be specified by the $\varepsilon$ kinetic energy functional form as follows:

$$
\bar{\varepsilon}_{\mathbf{k}}=\varepsilon_{\mathbf{k}}+I w_{1}(\varepsilon)
$$

For the pattern of this function at various temperatures, see Figure 11.

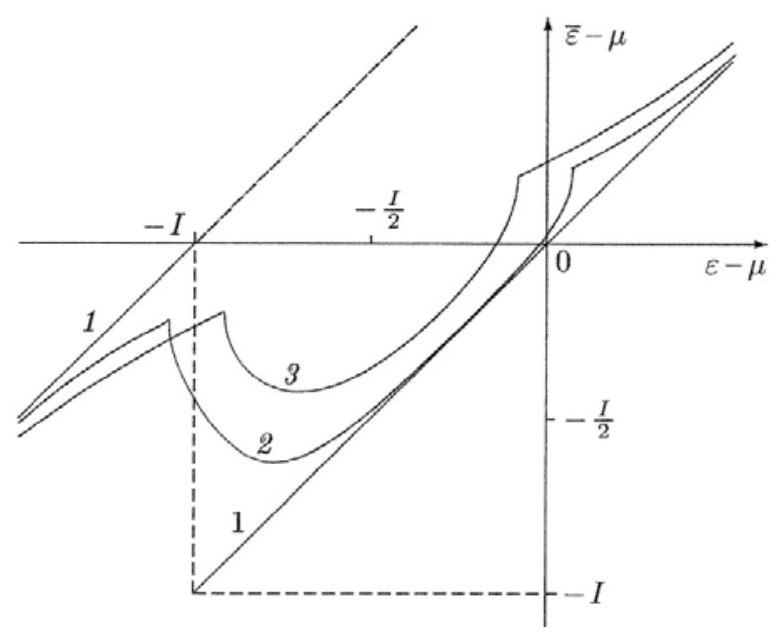

Figure 11. Mean electron energy $\bar{\varepsilon}$ dependence of its kineticenergy $\varepsilon$ at various temperature values: $1-\tau=0,2-\tau=0,5,3-\tau=0,8$ 


\section{Conclusion}

Thus, in scope of the theory discussed the microscopic superconductivity is due to anisotropic wave vector electron distribution. Normally, anisotropy is caused by electron repulsion effect in the $k$ and $-k$ wave vectors states and this is superconductivity. In conclusion we would like to say that the bulk of this performance lies in the formulas (1.5)

$$
\varepsilon_{\mathbf{k} \mathbf{k}^{\prime}}=I \delta_{\mathbf{k}+\mathbf{k}}{ }^{\prime}-J \delta_{\mathbf{k}-\mathbf{k}}{ }^{\prime}
$$

The basis for the proof of this formulas is the method of density matrices.

\section{References}

[1] H.Kamerlingh-Onnes, "Further experiments with liquid helium. C. On the change of electric resistance of pure metals at very low temperatures, ets. IV. The resistance of pure mercury at helium temteratures”. Comm. Phys. Leb. Univ. Leiden, (120b). 13-18. 1911.

[2] V.L. Ginzburg, L.D. Landau, "To the theory of superconductivity". JETF, 20, 1064-1071. 1950.

[3] J. Bardeen, L.N. Cooper, J.R. Schrieffer, "Theory of superconductivity". Phys. Rev., 108. 1175-1204.1957.

[4] J.R. Schiffer, Superconductivity Theory, (Nauka, Moscow, 1970).

[5] V.I.Bielawski,Y.V. Kopaev, "Superconductivity of repulsive particles". UFN, 176, 457-485, 2006.

[6] M.V. Sadowski, "High-temperature superconductivity in layerediron compounds”. UFN, 178, 1243-1271, 2008.

[7] B.V. Bondarev, "Quantum lattice gas. Method of density matrix", Physica A, 184.205-230.1992.

[8] B.V.Bondarev, "On some peculiarities of the electron distribution function Bloch states”, Vestnik MAI, 3 (2). 56-65.1 996.

[9] B.V. Bondarev, Density Matrix Method in Quantum Cooperative Process Theory, (Sputnik+, Moscow, 2013).

[10] B.V. Bondarev, Density Matrix Method in Quantum Theory of Superconductivity, (Sputnik+, Moscow, 2014).

[11] B.V. Bondarev, New Theory of Superconductivity. Method of Equilibrium Density Matrix. arXiv: 1412. 600822 Sep 2013.

[12] D.I. Blokhintsev, Principles of Quantum Mechanics, (Higher School, Moscow, 1961).

[13] Yu.I. Sirotin, M.P. Shaskolskaya, Basic Crystallophysics, (Nauka, Moscow, 1979). 